\title{
Dynamic Wicking Process in Textiles
}

\author{
Marcelo Parada ${ }^{1,4}$ (D) Peter Vontobel ${ }^{2}$. \\ René M. Rossi ${ }^{3}$ • Dominique Derome ${ }^{4}$ • Jan Carmeliet ${ }^{1,4}$
}

Received: 31 March 2017 / Accepted: 17 July 2017 / Published online: 4 August 2017

(C) Springer Science+Business Media B.V. 2017

\begin{abstract}
We investigate wicking in textiles using neutron radiography on fabrics of two types, woven and simple jersey knit, and made of yarns of four different materials: cotton, polyethylene terephthalate, polyamide and polypropylene. Moisture distribution in the samples is quantified from the images, and the total mass is compared with gravimetric measurements for validation. The position of the front versus time is obtained from segmented neutron images and backlight images, with good agreement between the two measurement methods. Using a power law to fit the height versus time curves, values for the wicking ratio are found to depart substantially from the 0.5 predicted by the Lucas-Washburn equation. The sharp front approximation is found to be a good approximation for slow wicking fabrics but is an incomplete description of the moisture on high absorbing fabrics such as cotton. The variation of yarn material is the main contributor to the difference of wicking ratio among samples. Finally, neutron moisture profiles complemented with X-ray tomography allow to differentiate two regions in cotton wetted by wicking: intra-yarn wetting and wetting covering the yarns and the inter-yarn voids.
\end{abstract}

Keywords Wicking $\cdot$ Neutron radiography $\cdot$ Moisture quantification

\section{Introduction}

Spontaneous liquid imbibition due to capillary forces occurring in a mainly two-dimensional system is called here wicking. Capillary forces arise in the presence of a hydrophilic liquid-

Marcelo Parada

marcelo.ito.parada@arch.ethz.ch

1 Institute of Technology in Architecture, Chair of Building Physics, ETH Zurich, Stefano-Francini-Platz 1, 8093 Zürich, Hönggerberg, Switzerland

2 Neutron Imaging and Activation Group, PSI, Villigen, Switzerland

3 Laboratory for Biomimetic Membranes and Textiles, EMPA, St. Gallen, Switzerland

4 Laboratory for Multiscale Studies in Building Physics, EMPA, Dübendorf, Switzerland 
solid contact angle, pulling the liquid into the pore or void space. For this situation to occur, in addition that the solid must be wettable by the liquid, the geometry must allow the formation of a meniscus (Masoodi and Pillai 2012). Textiles are multi-scale porous materials, whose pores can be understood to be the free space between the intertwined yarns as well as the free space between the fibers of each yarn. In effect, the textile yarns are formed of twisted or spun fibers, from a few to a hundred per yarn. These fibers can be of different materials and have different geometrical cross sections and physical properties. Yarn parameters such as diameter, twisting level and fiber density can influence wicking. At the next scale of complexity, a textile fabric consists of yarns interlaced most often in a woven or knit structure, although other arrangements are also possible. Textile directions are named as follows: warp and weft, respectively, perpendicular to and along the weaving direction for woven, and course (path of yarn) and wale for knits. Wetting direction, textile structure and density and yarn count can influence wicking. Wicking can occur in different situations where the textile is exposed to a liquid; however, understanding wicking in textiles is particularly important for situations where inappropriate moisture management can lead to injuries such as heat burns in firefighters (Keiser et al. 2010) and decubitus in immobile patients (Derler et al. 2012; Rotaru et al. 2013; Campbell and Parish 2010).

The first step to understand wicking is to accurately measure or quantify this phenomenon. Many studies have investigated wicking using visible light directly (e.g., Raja et al. 2014; Taheri et al. 2013; Vatansever et al. 2012) or using changes in color, lightness or reflectance (Lee et al. 2004). However, visible light can only illuminate the surface and changes in moisture content that do not occur at or near the surface may go undetected with such surface measurement. When colored water is used, the colorants can affect the liquid surface tension or viscosity and, thus, its wicking behavior. Infrared radiation imaging measuring surface temperature used to image wicking (Niedermann and Rossi 2012) is also limited to imaging the surface. Contact measurements using electrical impedance (Hu et al. 2005; Yao et al. 2006; Wardiningsih and Troynikov 2012) or thermo-couples (Zhu and Takatera 2014, 2015) can measure moisture or temperature across the textile but the necessary contact may locally affect water transport. Finally, X-ray imaging has been used to document wicking by Birrfelder et al. (2013), Weder et al. (2006), allowing localization of moisture and even the determination of the three-dimensional configuration of water. Also Birrfelder et al. (2013) were able to recognize waterfronts that could be interpreted as distinct intra-yarn and interyarn in-plane wicking fronts. However, the absorption contrast of light elements (such as water molecules) is low. This can be mitigated by the use of a contrasting agent (Weder et al. 2006) or using phase contrast techniques [an example, although not on textiles, can be seen in Eller et al. (2011)]. In spite of these advances, quantification of moisture content is still challenging based on X-ray images. A more complete review of imaging techniques applied to wicking can be found in Parada et al. (2017) which indicates that one imaging method can provide the necessary quantification of moisture content within the material. Neutron radiation is sensitive, among others, to hydrogen resulting in good contrast to water molecules (Pleinert and Lehmann 1997), and neutron radiography has successfully been used to quantify moisture content in textile materials during drying (Reifler 2006). Here we will use neutron radiography to document wicking as neutron radiography yields quantified moisture content not only globally for the whole sample, but also locally for each image pixel.

In this paper, we consider first the question as to whether neutron radiography can be applied for the quantitative measuring of wicking. We present the image processing necessary for the quantification, and we compare the results to the classical visual light technique and to gravimetry. The second question addresses the fact that wicking in fabrics has been 
classically evaluated as a two-dimensional process where the behavior of the wicking front or area is believed to be sufficient to describe the phenomenon. Since neutron radiography yields the moisture content distribution over the whole fabric, we can investigate whether the two-dimensional approximation of a front is an adequate approach. Finally we investigate different materials and fabric structures to capture which parameters affect wicking and in which situations the two-dimensional front approximation could be appropriate.

In the next section, we describe the materials, the sample holders as well as the experimental procedure and setup. Then, in Sect. 3, we provide first details about the neutron radiography method, both in terms of acquisition and moisture content quantification, followed by details regarding the backlighting method, and the X-ray tomography method used to verify the tridimensional configuration of water. In Sect. 4, we show the image analysis procedures to go from raw neutron radiography images to moisture content distribution fields and to identify the wicking front at every image. Then we compare the results of backlight imaging with the results from neutron radiography. With the whole procedure confirmed, experimental results for the different fabrics as well as the analysis of the wicking behavior are presented in Sect. 5. Finally, we draw our main conclusions.

\section{Materials and Methods}

\subsection{Materials}

We use yarns of four different materials - polyethylene terephthalate (PET), polyamide (PA), polypropylene (PP) and two types of natural cotton (brown and white) - at 2 or 3 different twisting levels each. PP yarns present different fiber cross sections. Table 1 lists the configuration and properties of the yarns as provided by the manufacturers. Yarns of 2 or 3 different twisting levels could be acquired directly from the manufacturers, except for cotton where only a twist level of $800 \mathrm{tpm}$ was available. So some cotton yarns were retwisted to a level of $1000 \mathrm{tpm}$. The contact angles were measured by taking images of the yarn dipped perpendicularly in a water reservoir and measuring the angle on both sides of the projection with the Fiji distribution of ImageJ (Schindelin et al. 2012). At least three images of each yarn were captured, and the values were averaged across all twisting levels and cross sections.

The yarns were knitted in a circular knitting machine (simple jersey) and woven (plain weaving) in a manual mechanical loom to produce 13 different knitted and 10 different woven fabrics. For technical reason, the warp yarn of all woven samples was PET. The 13 knitted samples were tested in both course and wale directions. The 10 woven samples were tested only in weft direction because the warp thread for all samples was the same PET thread. One (PET) woven sample was also tested in the warp direction. The PP knit fabrics of different cross sections (tetra channel, triangular and hollow circular) were only tested in the course direction. Before testing, the samples were washed three times at $40{ }^{\circ} \mathrm{C}$ to remove any trace of yarn fabrication residuals. A list of the fabrics tested can be found in Table 2 .

In order to avoid curling due to swelling, a sample holder is used to maintain the fabric flat and perpendicular to the beam in backlight and neutron imaging. Two mounting methods were used: in the first version, the fabrics were cut to be at the full dimension of the holder, i.e., $70 \times 100 \mathrm{~mm}^{2}$ and assembled in sandwich between the two holder frames (Fig. 1a, left). In the second method, the fabrics were cut to smaller dimensions of $50 \times 80 \mathrm{~mm}$ and the edges were stitched to avoid shredding (Fig. 1a, right). They were then mounted in the center of the sample holder using a hydrophobic PET thread. The second version of the sample 


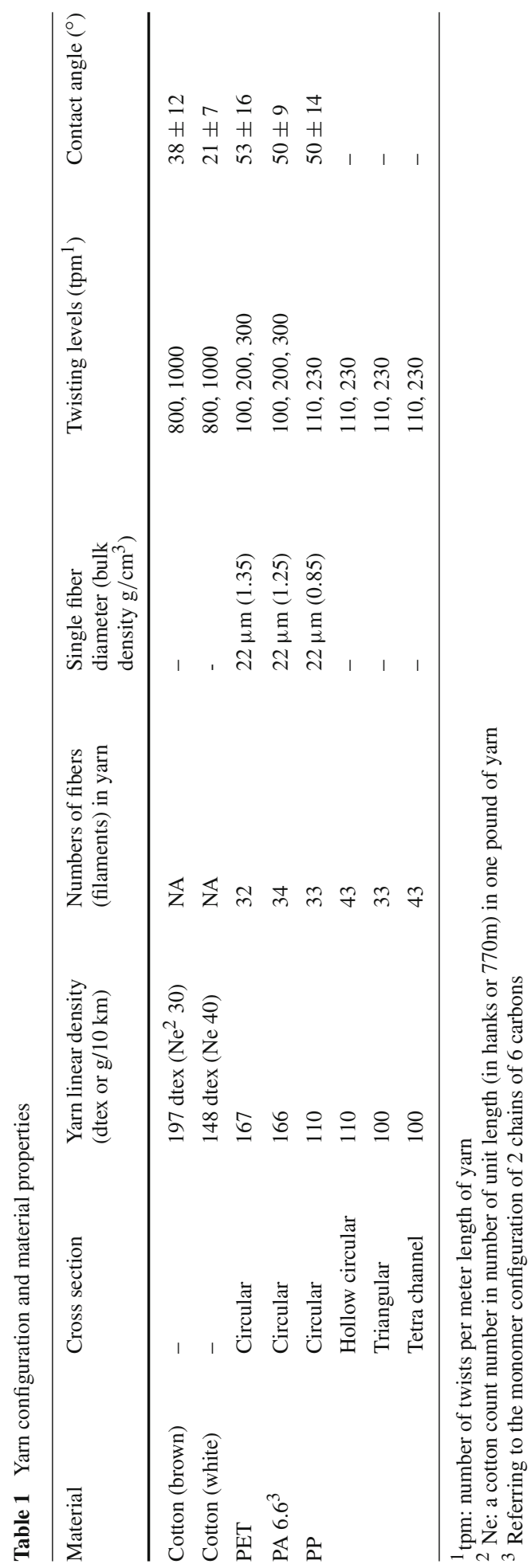


Table 2 Tested fabrics

\begin{tabular}{|c|c|c|c|c|}
\hline \multirow[t]{2}{*}{ Yarn, twist, cross section } & \multicolumn{2}{|c|}{ Structure } & \multicolumn{2}{|c|}{ Directions tested } \\
\hline & Knit & Woven & Course, wale & Weft \\
\hline Cotton (brown), 800 & $\mathrm{x}$ & $\mathrm{x}$ & $\mathrm{x}$ & $\mathrm{x}$ \\
\hline Cotton (brown), 1000 & $\mathrm{x}$ & $\mathrm{x}$ & $\mathrm{x}$ & $\mathrm{x}$ \\
\hline Cotton (white), 800 & $\mathrm{x}$ & $\mathrm{x}$ & $\mathrm{x}$ & $\mathrm{x}$ \\
\hline Cotton (white), 1000 & $\mathrm{x}$ & $\mathrm{x}$ & $\mathrm{x}$ & $\mathrm{x}$ \\
\hline PET, 100 & $\mathrm{x}$ & $\mathrm{x}$ & $\mathrm{x}$ & $\mathrm{x}$ \\
\hline PET, 200 & $\mathrm{x}$ & $\mathrm{x}$ & $\mathrm{x}$ & Weft and warp \\
\hline PET, 300 & $\mathrm{x}$ & $\mathrm{x}$ & $\mathrm{x}$ & $\mathrm{x}$ \\
\hline PA, 100 & $\mathrm{x}$ & $\mathrm{x}$ & $\mathrm{x}$ & $\mathrm{x}$ \\
\hline PA, 200 & $\mathrm{x}$ & $\mathrm{x}$ & $\mathrm{x}$ & $\mathrm{x}$ \\
\hline PA, 300 & $\mathrm{x}$ & $\mathrm{x}$ & $\mathrm{x}$ & $\mathrm{x}$ \\
\hline PP, 110 , circular & $\mathrm{x}$ & & Only wale & \\
\hline PP, 230, circular & $\mathrm{x}$ & & $\mathrm{x}$ & \\
\hline PP, 230, hollow circular & $\mathrm{x}$ & & Only course & \\
\hline PP, 230, tetra channel & $\mathrm{x}$ & & Only course & \\
\hline $\mathrm{PP}, 230$, triangular & $\mathrm{x}$ & & Only course & \\
\hline
\end{tabular}

holder was designed to separate the samples from the frame to avoid edge effects in slow wicking materials. Both sample holder versions were made of aluminum for its low neutron attenuation. As mentioned above, the fabrics were assembled with weft direction along the longer direction of the holder for woven structure, and both course and wale directions for knitted structure. Also, a smaller holder was devised to hold the sample during X-ray tomography, where the textile specimen is glued to a U-shaped polymethylmethacrylate frame of $25 \times 75 \mathrm{~mm}^{2}$, as shown in Fig. $2 \mathrm{a}$.

The liquid used for almost all experiments is deionized water. The exception is the liquid used for the X-ray CT images. For the CT images a 10\% vol solution of polyoxyethylene (20) sorbitan monolaurate (Tween 20, Sigma-Aldrich product number P2287) was used. The concentration of the surfactant in the solution is above the critical micelle concentration so its surface tension is constant at $35 \mathrm{mN} / \mathrm{m}$ (Mittal 1972; Niño and Patino 1998). Contact angle of cotton yarns with the surfactant solution is $0^{\circ}$, and density change is negligible.

\subsection{Experimental Setup and Procedure}

The wicking test setup consists of a balance, a support for the sample that is resting on the balance and another support that carries an electric motor that activates a lift carrying the water reservoir. This second support is independent from the balance. A schematic and a photograph of the setup can be seen in Fig. 1c, d.

The images during wicking are taken with two methods (not concurrently): neutron radiography and backlighting, explained in detail below. Once installed for proper image acquisition, the sample is first imaged in dry state. The dry sample did not have any specific conditioning prior to the measurement. Next, the reservoir is brought up to the sample and wicking starts with regular interval imaging. After the water front reaches the top of the sample or does not perceptually move anymore, the reservoir is brought down and final images are taken of the 

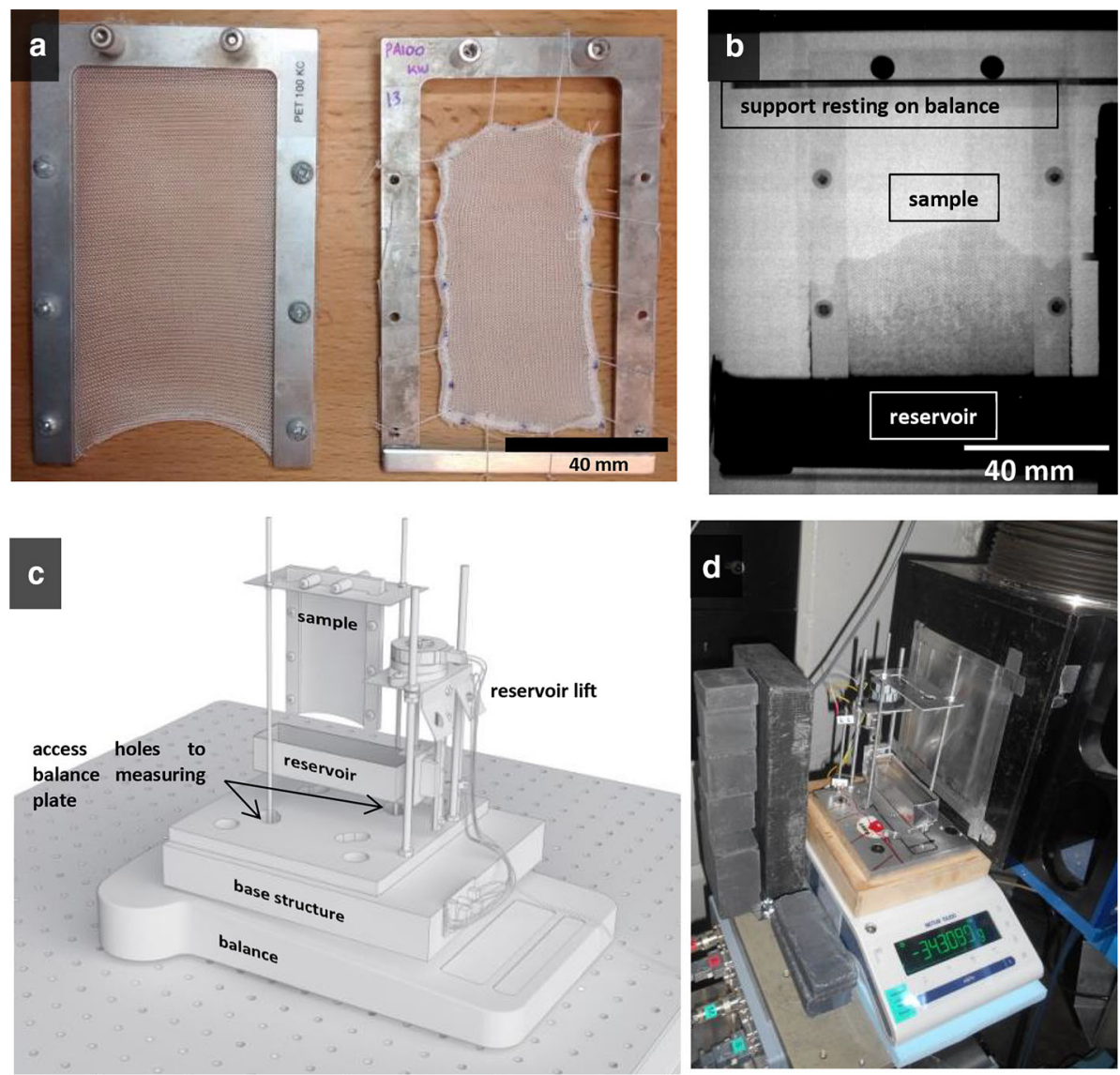

Fig. 1 a Sample holders version 1 (left) and 2 (right). b Example of a raw image of neutron radiography of cotton knit sample undergoing water wicking. c Setup schematic showing the sample, the reservoir, the reservoir electric lift, the base structure and the balance. The reservoir and its lift are supported by two pillars (on both sides of the lift) attached to the base which rests on the balance casing (but not on the measuring plate). The sample is supported by two pillars that reach the measuring plate of the balance through two access holes in the base. $\mathbf{d}$ Photograph of the setup inside the NEUTRA beamline, with boronated polyethylene blocks on its left to protect the electronics from the neutron beam, and with the scintillator on its right. The neutron beam crosses from left to right

fully wet sample without the reservoir on the field of view. The water level at the reservoir has no discernible change throughout the experiment. The reservoir movement is remotely controlled to permit repeatability and avoid sample displacement. Care is taken in keeping the sample immobile throughout the experiment to simplify the subsequent image processing (see Sect. 4: Image Processing).

The whole experiment is performed with the sample resting on the balance, and its increasing mass digitally monitored. Moreover, a logger continuously records air temperature and relative humidity in the experimental chamber. In the first set of neutron radiography experiments (using sample holder version one), the temperature was $26.2 \pm 0.4{ }^{\circ} \mathrm{C}$ and the relative humidity $48 \pm 6 \% \mathrm{RH}$, while in the second set of neutron radiography experiments (sample holder version two), the values were $26.7 \pm 0.7^{\circ} \mathrm{C}$ and $39 \pm 6 \% \mathrm{RH}$, respectively. Not 

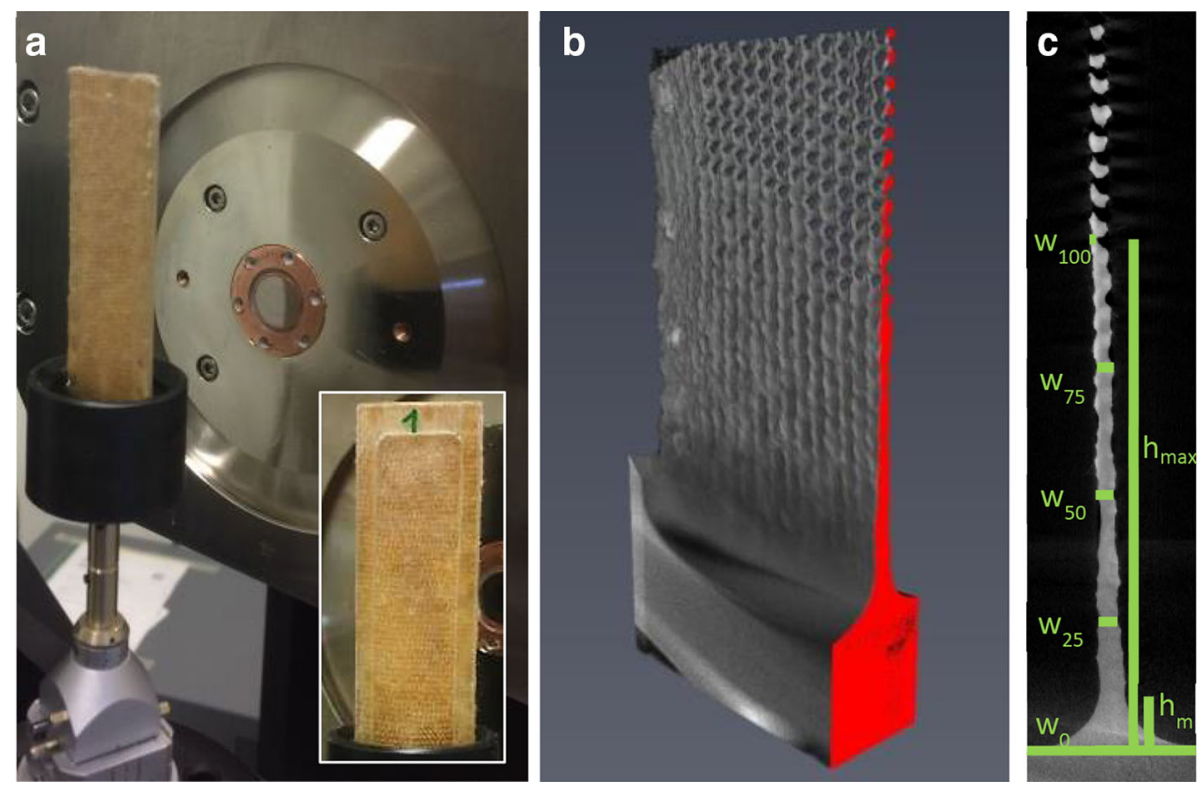

Fig. 2 a Photographs of the setup on the X-ray CT stage, insert shows the $U$-shaped sample holder. b Cut perspective of X-ray tomography reconstruction of the wet cotton sample at the end of wicking, with the water reservoir at the bottom. c Cross section of tomography showing lower wicking region. Marked in green: $h_{\max }$ height of lower region, $h_{m}$ height of meniscus, $w$ width at $0,25,50,75$ and $100 \%$ of height

all samples were tested in both experimental setups. Since no difference was seen between the two sample holders, the results presented in this paper are aggregated from both sets of experiments.

\section{Imaging Approaches}

\subsection{Neutron Radiography}

Neutron radiography is a non-destructive imaging technique that uses a neutron beam as radiation. Similar to X-ray radiography, the beam traversing the sample interacts with the sample and a two-dimensional (2D) projection of the transmitted beam is recorded by a detector. Unlike X-rays that interact with the electron shell of an atom, neutrons interact with the nucleus. As a result, the level of interaction is not linked to the atomic number. In particular, neutrons interact strongly with hydrogen, resulting in high contrast when imaging water (Lehmann et al. 2004; Sedighi-Gilani et al. 2014; Derluyn et al. 2013). This high sensitivity to water allows an accurate quantification of moisture content in the sample.

Our measurements were performed at the Neutron Transmission Radiography (NEUTRA) beamline at the Swiss Spallation Neutron Source (SINQ) of the Paul Scherrer Institute (PSI) in Villigen, Switzerland. The conical neutron beam is controlled by a collimator, and the sample is situated at a distance from the collimator yielding a final field of view of $107 \times 133$ $\mathrm{mm}^{2}$. The beam traverses the sample and, as it meets the $100-\mu \mathrm{m}$-thick zinc sulfide scintillator doped with ${ }^{6} \mathrm{Li}$ as the neutron absorbing agent, it is transformed in visible light, which is photographed with a sCMOS (Andor Neo) detector system of $2560 \times 2160$ pixels. The images 
are binned $2 \times 2$ resulting in $1080 \times 1280$ pixels. The system yields a pixel size of $104 \mu \mathrm{m}$ at 16-bit dynamic range. More details of the facility are available in Lehmann et al. (2001). The beam is polychromatic within the thermal spectrum (Lehmann 2008) and the treatment of information is spectral. For simplicity, we present the moisture content quantification processing of the images using the equations for a monochromatic beam. After interaction with the sample, the intensity of the transmitted beam can be described by the Beer-Lambert law:

$$
I=I_{0} e^{-\Sigma \cdot z}
$$

where $I_{0}$ is the intensity of the incoming beam (in Watts), $z$ is the length of the material along the beam direction or sample thickness (in $m$ ) and $\Sigma$ is the linear neutron attenuation coefficient of the sample (in $1 / m$ ). For compound materials, each compound can be considered to have a thickness, from 1 to $i$, and Eq. (1) becomes:

$$
I=I_{0} e^{-\Sigma_{1} \cdot z_{1}-\Sigma_{2} \ldots z_{2} \ldots-\Sigma_{i} \cdot z_{i}}
$$

For our specific case, we separate the sample in the beam field of view (FOV) into two: the dry material, $d$, and water, $w$ :

$$
I=I_{0} e^{-\Sigma_{d} \cdot z_{d}-\Sigma_{w} \cdot z_{w}}=I_{0} e^{-\Sigma_{d} \cdot z_{d}} e^{-\Sigma_{w} \cdot z_{w}}
$$

We note that $I_{\mathrm{dry}}=I_{0} e^{-\Sigma_{d} \cdot z_{d}}$ is the intensity obtained in the detector for the initial dry images. The water thickness for a given image can then be obtained as follows:

$$
z_{w}=\frac{1}{\Sigma_{w}}\left(\ln \left(I_{\mathrm{dry}}\right)-\ln (I)\right)
$$

From the water thickness, water density and pixel size, we calculate the water mass at each pixel:

$$
m_{w}=z_{w} \cdot \Delta_{x}^{2} \cdot \sigma_{w}
$$

where $\Delta_{x}$ is the pixel size (in our specific case $\Delta_{x}=104 \mu \mathrm{m}$ ) and $\sigma_{w}=997 \mathrm{~kg} / \mathrm{m}^{3}$ is the water density.

The field of view of $110 \times 130 \mathrm{~mm}^{2}$ allows imaging the whole sample (including the sample holder) and part of the support structure and the reservoir (see Fig. 1b), while the rest of the setup is protected with boronated polyethylene blocks. The exposure time chosen was $3 \mathrm{~s}$ for the cotton samples and $10 \mathrm{~s}$ for all other samples. These values are a good compromise between the signal strength and the motion artifacts, resulting from the motion of the water front during the acquisition.

\subsection{Backlight Imaging}

The same samples and setup were also imaged using a backlight imaging technique. The illumination is visible light and the image recorded is the transmitted light. Illumination is provided by a LED light of 7700 lumens (Videal MultiLED LT), and the images are recorded by a CCD camera (Nikon D90). The field of view used is $127 \times 85 \mathrm{~mm}^{2}$, with a pixel size of $60 \mu \mathrm{m}$ for a detector of $2144 \times 1424$ pixels (RGB, 8-bit). Images were recorded with a frequency of 1 image per second. 


\subsection{X-ray Microtomography}

As a complementary documentation of the water configuration on the textile, we perform one tridimensional (3D) X-ray imaging. A sample of brown cotton knit, smaller than the samples imaged with backlight and neutron imaging, was imaged in steady wet state, i.e., once wicking front reached steady state, with a laboratory X-ray computer tomography scanner (CT). The anode voltage was set at $50 \mathrm{kV}$. 900 projections were taken over $360^{\circ}$. Each projection was the average of 4 images, each with $500 \mathrm{~ms}$ acquisition time. Adding software and motor latency, the total time for each projection was $4 \mathrm{~s}$, resulting in a total scan time of one hour. Since the projections could be reliably reconstructed to 3D images, there was no significant change in the geometry during the measurement (i.e., negligible effect of evaporation or movement). The images were captured by a CCD detector cropped to $1800 \times 2028$ pixels. Since the beam has a cone geometry, some edge pixels are lost in the reconstruction. The final 3D reconstructed volume has $335 \times 1307 \times 1829$ voxels of $17.96 \mu \mathrm{m}$ per side. A cut view of the resulting 3D dataset is achieved using the Avizo software and can be seen in Fig. 2b.

\section{Image Processing}

\subsection{Moisture Content Quantification}

The images obtained by neutron radiography are intensity images, which can be converted to obtain the water thickness at each pixel as presented in Sect. 3.1. The quantification is done using the Quantitative Neutron Imaging (QNI) software tool (Hassanein 2006) which also corrects for the polychromatic nature of the beam, dark current, flat field and noise. In short, the tool removes extreme outliers using a median filter, corrects for inhomogeneous illumination and background noise, corrects for beam hardening and finally applies the Beer-Lambert law to obtain the mass thickness of water in every pixel. More details of the quantification procedure can be found in Sedighi-Gilani et al. (2012). Note that for scattering we do not correct using QNI which also provides this feature, but use a different approach as explained next.

\subsection{Correction of Scattering from the Reservoir}

A main source of neutron scattering in this experiment is the water in the reservoir, which has a water thickness of one or two orders of magnitude larger than the one of the wet fabric. Scattering effectively results in a skew toward higher values close to the reservoir as seen in Fig. 3, an effect probably due to the geometry of the reservoir. We correct for this scattering by finding the difference between images with and without the reservoir. At the end of the wicking experiment, we record a few images without the reservoir to be used for comparison with the images taken immediately before the reservoir removal. The scatteringinduced difference of moisture thickness is fitted with a curve over a height of 500 pixels (52 $\mathrm{mm}$ ) above the reservoir level, after which there is no significant scattering. The fitted curve is smoothed along the height using a Savitzky-Golay method (Savitzky and Golay 1964; Schafer 2011) of the eighth degree over a 101 pixel span. This smoothed-fitted curve is used to correct all the images of each wicking experiment. 


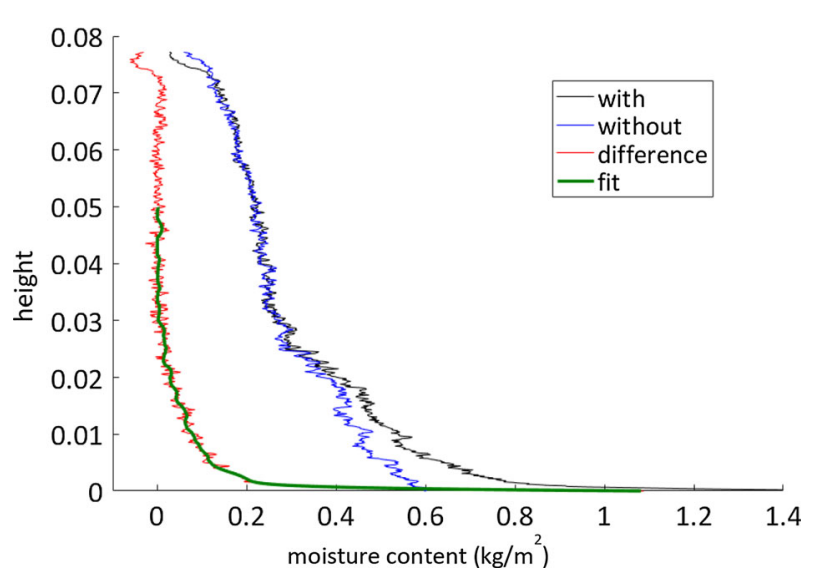

Fig. 3 Moisture content profiles along the height of one sample, with and without the reservoir in the field of view, in red is the scattering-induced difference and in green is the fit used in further image processing

\subsection{Image Analysis}

A stack of 10 initial dry images, thus before initiation of water uptake, are averaged pixelwise, and this average image is used as dry reference image. This reference is then subtracted from all other images in the dataset. This step actually removes the contribution of all elements in the images which are not water. As mentioned before, the samples remained immobile throughout the experiment, making this subtraction effective and simple as no registration or alignment was necessary. We note that images are subtracted and not divided because they are already quantified, i.e., all images are readily mapped from intensity to mass thickness with Eqs. (3)-(5). The reference image is also mapped to an equivalent thickness to allow for this subtraction.

\subsubsection{Front Finding}

To obtain the position lines of the water front in terms of height versus time, the water front is identified in all images using the following steps: 1. Gaussian filter, 2. threshold, 3. labeling, 4. geodesic transform and 5. front identification.

First, we apply a Gaussian filter to reduce noise and the influence of the textile own structured pattern. Then we determine a suitable threshold $T$ by picking a threshold value lying in between the wet and dry areas of an arbitrary image using Eq. (6). The parameter $\alpha$ used is a compromise between a low value (close to zero) which detects too much noise and a high value (close to 1 ) that fails to detect all wet regions of the fabric. A value of 0.3 is found to be appropriate for most fabrics. For the few ones for which this parameter was not suitable (as visually assessed) a value was chosen manually between 0.1 and 0.8 . This threshold is then used for all the images in an uptake sequence. We define the samples as initially dry.

$$
T=\alpha\left(m_{\text {wet }}-m_{\text {dry }}\right)+m_{\text {dry }}
$$

After thresholding, we are left with a binary image. This image is very noisy, as can be seen with an example in Fig. 4a. Part of the noise is removed by a closing morphological operation, but the largest noise patches cannot be removed this way. Therefore, we label all 

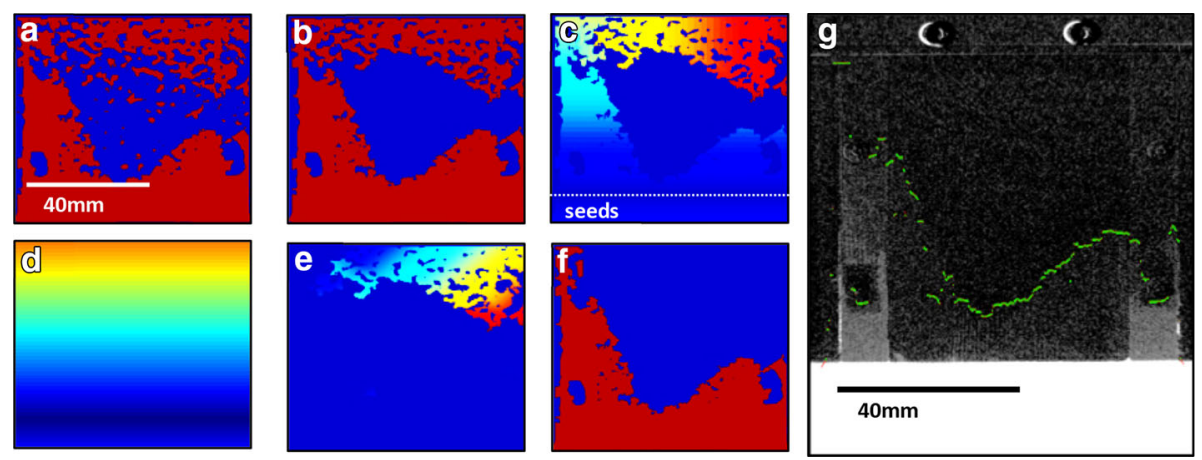

Fig. 4 Front finding processing of binary images of a PET sample. Images obtained a after thresholding. b After labeling and selection of the region connected to reservoir. c Geodesic transform. Seeds are in the horizontal line inside reservoir (dashed white line). $\mathbf{d}$ Template of geodesic transform. e Regions where images differ greatly from template. f Final segmentation. g Identified front (in green) superimposed to the starting subtracted image

contiguous regions and ultimately keep only the region which includes the reservoir (see Fig. 4b).

At this point, in most cases, the wet area is already clearly defined. In some cases, however, we may find a noise patch that "touches" the wet part and is misidentified. To correct for that, we calculate the geodesic distance transform from seeds placed in a horizontal line inside the reservoir (see Fig. 4c). In our case, we used the Manhattan distance function, Eq. (7), for the geodesic distance:

$$
d(x, y)=\left|x_{1}-y_{1}\right|+\left|x_{2}-y_{2}\right|
$$

At the same time, we calculate a template of the geodesic transform for a fully wet image (see Fig. 4d). Finally we subtract the image from the template and discard any pixels where the difference is larger than a given value (here, 10 pixels). Figure $4 \mathrm{e}$ shows the pixels that would be discarded for the example. The final result of this processing can be seen in Fig. $4 \mathrm{f}$. To identify the front, we simply find the first nonzero pixels of the binary image from the top for each column as applied in the example image (Fig. 4g). We determine the height of the front as the average height of 200 pixels $(21 \mathrm{~mm})$ in the middle of the sample. We do not use the whole sample to avoid edge effects. Note that a front could also be tracked inside the frame with neutron radiography, if deemed of interest in some other application.

Figure 5 shows the same image processing technique applied to a cotton sample. Due to the larger absorption of moisture in cotton, the signal is much stronger and the front identification is easier. The geodesic template and the seeds used were the same for all samples.

\subsection{Comparison of Front Tracking in Neutron and Backlight Imaging}

To validate the moisture distribution and the segmentation procedures applied on the neutron radiography, one sample of PA was tested three times in our wicking setup using the backlight technique. Backlight images are not penetrating images; thus, it is not possible to quantify the water in the images. It is possible, however, to detect the wicking front. The image analysis procedure is analogous to the neutron images (Sect. 4.3). First, the 10 initial dry images are averaged and used as a dry image reference. Second, the dry image reference is subtracted from all images in the time series to obtain only the wet part of the images. Note that, at this 

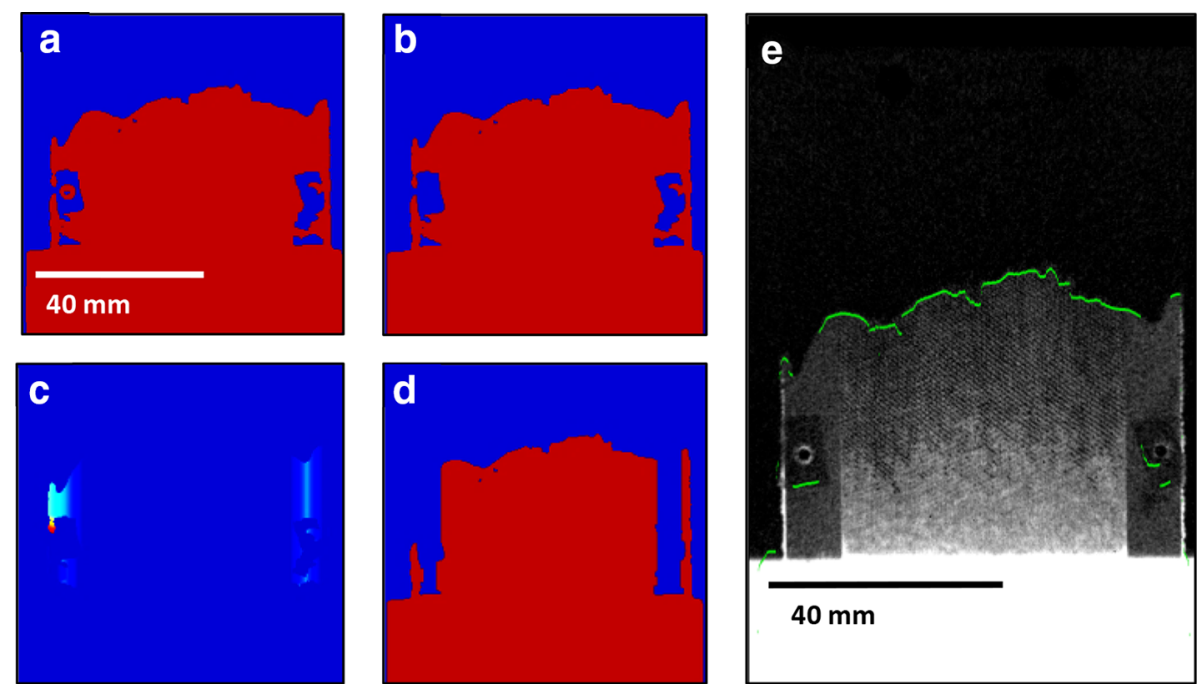

Fig. 5 Processing of binary images of a cotton sample. Images obtained a after thresholding. b After labeling. c Regions identified by the geodesic transform as different from template. d Final segmentation. e Resulting front (in green) superimposed to the starting subtracted image
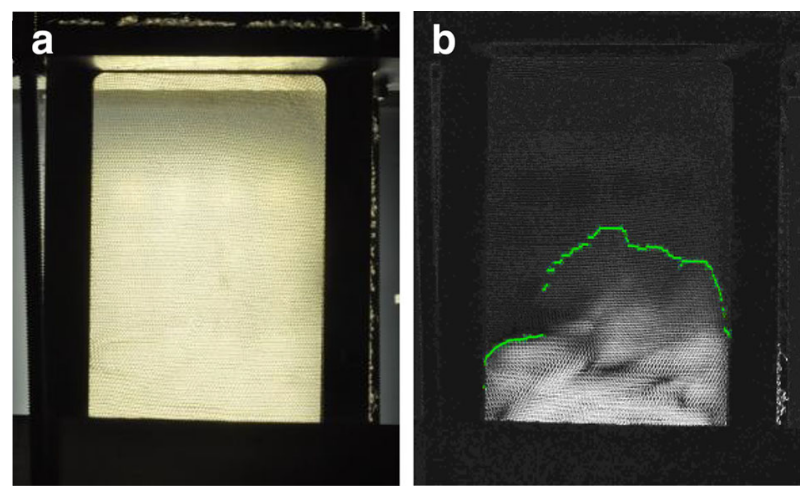

Fig. 6 a Sample image of backlighting technique (left) and b subtracted image with identified front (right) at an arbitrary time step during wicking. The wet region can only be seen by eye after the subtraction step

step, the images are converted from RGB, 8-bit, to 16-bit to allow for a larger intensity range. Finally the front is identified in the subtracted images with the same procedure as the one used for the neutron images (Sect. 4.3.1). The main difference in this final step is the threshold value. A fixed threshold value of 5 was defined as a good compromise between reliably detecting the front and good signal-to-noise ratio. Figure 6 shows an image as recorded by the camera and the identified front superimposed on the subtracted image at an arbitrary time step. As done above, front height is defined as the average front height in the middle $21 \mathrm{~mm}$ of the sample.

The results of front height versus time from three backlight and one neutron imaging experiments are shown in Fig. 7. It is clear there is variability in wicking behavior even for the same sample, but the overall behavior can be captured by both imaging techniques. The backlight measurements stop at the top of the visible section of the textile, while the neutron 


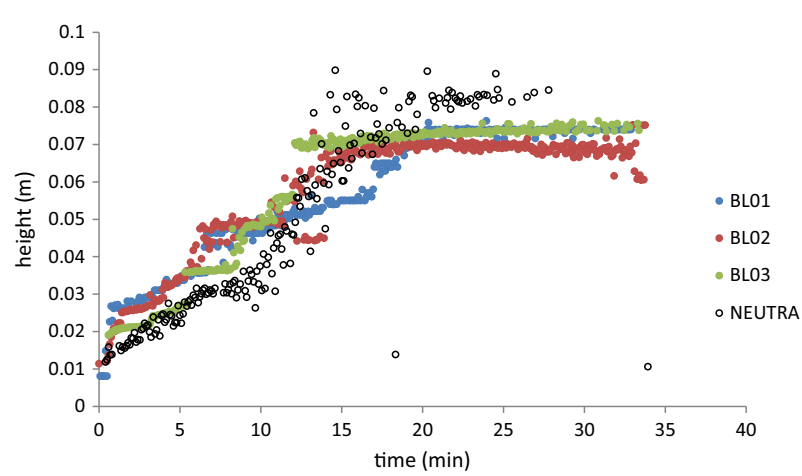

Fig. 7 Comparison of front position height versus time between neutron radiography (white) and repeats of backlight imaging (color) of a single PA sample

radiography measurements follow the wicking in the textile even passed the aluminum top parts of the frame.

\section{Results and Discussion}

We analyzed the performed neutron experiments in terms of total mass versus time, moisture mass distribution and profiles versus time and front height versus time.

\subsection{Total Mass Versus Time}

The neutron radiography technique allows us to investigate the total moisture uptake into the fabric during wicking as well as the moisture content distribution over the specimen. The gravimetric method only provides information of the aggregate amount of moisture in the sample versus time. We use the gravimetric results to calibrate the moisture content as determined from the neutron images. By using one scaling factor per sample, we achieve good agreement between the total moisture content in the sample as measured by the balance and as imaged with the neutron beam, and this for all samples except for the PP fabrics where the amount of water absorbed was too low to gather reliable data.

Figure 8 shows the total moisture content measured for different samples versus time. For cotton (Fig. 8a), there is good agreement between the values obtained through the neutron images and the values obtained by the balance. The PA (Fig. 8b) and PET (Fig. 8c) samples, which have a much lower absorption of moisture as shown by the different $y$-axis ranges, display less agreement between measurements from the two techniques. The overall behavior, however, follows the same tendencies. The same mass plots can be visualized in logarithm scale, as shown in the right column of Fig. 8. The mass measurements with the balance and with the neutron images display similar slopes, although some shifts are observed. As a first observation, the main variable affecting wicking is the material from which the yarns are made. Some parameters which were not controlled for such as finish can also have a big impact.

In terms of effects of textile structure and direction, and of effects of yarn twisting levels, we cannot observe any clear trend. The parameters investigated were: material, twist level, fabric structure, direction and fiber cross section. Only the material showed significant change. Cross sections could not be evaluated because the PP fabrics, for which we had different cross sections, did not wick enough for good contrast. 

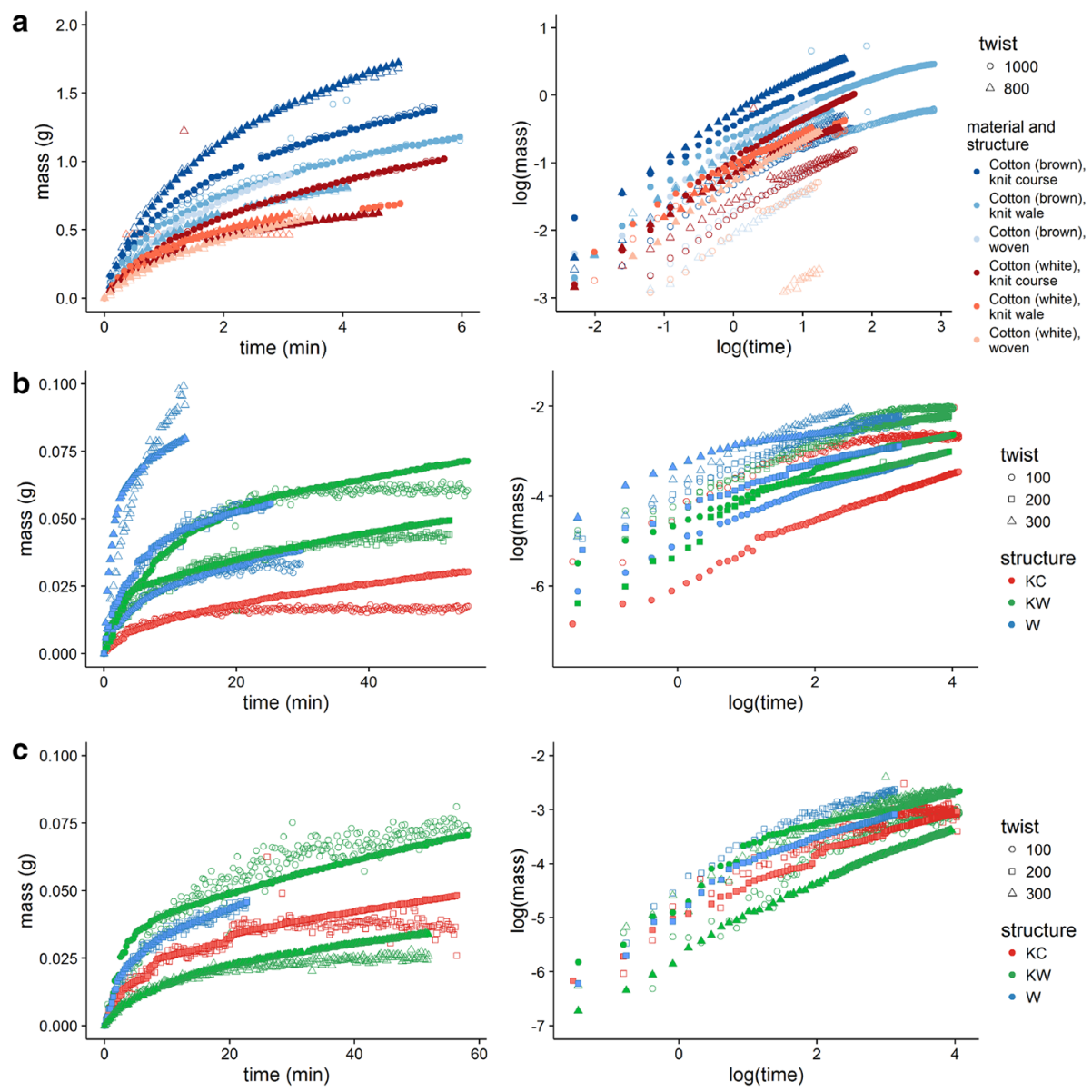

Fig. 8 Total moisture content as measured by the balance and the images in linear (left) and log (right) scales. Balance measurements are solid; neutron image measurements are hollow. Results are for a cotton samples, b PA samples, c PET samples. Note the different uptake durations for the different materials. $K C$ knit course, $K W$ knit wale, $W$ woven

\subsection{Moisture Maps}

A good visualization of the moisture distribution can be achieved with moisture maps based on neutron images. The pixel intensity or color indicates the moisture content of the pixel (i.e., local moisture content). Figure 9 shows moisture maps, each taken at selected time steps (different for each sample), for selected samples of textiles of different materials. The dark red part in the bottom is the reservoir. As can be seen, the amount of moisture absorbed by the cotton sample is much higher than what is observed in the synthetic samples. Wicking in PA and PET results in low moisture content, difficult to see by eye, but sufficient for quantification as can be seen later in the moisture profiles of Fig. 10. The PP sample, however, clearly shows that wicking is not occurring in the fabric.

Figure 11 shows the moisture maps evolution for a knitted cotton sample at 12 different time steps. We note that the fronts are approximately flat across the sample at all times. There 

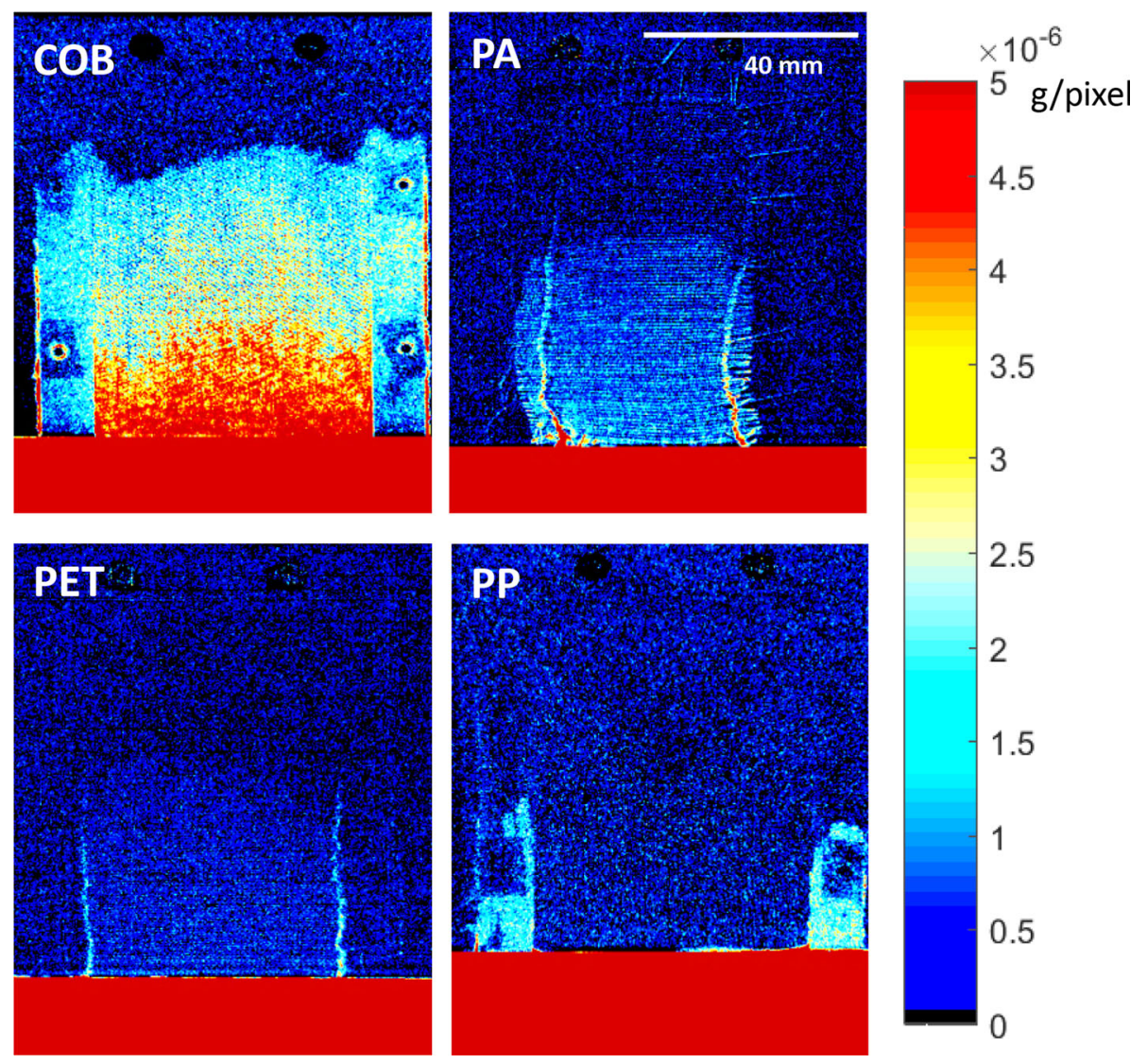

Fig. 9 Typical moisture distribution maps textile samples made of the different materials. Color scale represents moisture content in each pixel. Red region at the bottom is the water reservoir

are two regions developing over time: one in the bottom with large moisture content (above $0.4 \times 10^{-5}$ g per pixel or red) and one in the top with lower moisture content shown as blue. The bottom region appears stable after reaching a height of approximately $20 \mathrm{~mm}$, while the higher region continues to increase until it reaches the top of the sample. The knit pattern is visible in the top region, while the lower region is homogeneous and presents no distinct pattern. This suggests that the moisture in the top region is present mainly inside the yarns, with the voids between the yarns remaining empty and thus allowing for the structure of the textile to be displayed. The lower region, with higher moisture content, contains moisture bridges between the yarns as well as inside the yarns, hence the appearance of a rather continuous film of water.

\subsection{Moisture Distribution in Two Regions for Cotton Sample}

Such splitting into two regions of the moisture distribution during wicking warrants the introduction of the third imaging technique used in this investigation, namely X-ray microtomography. We thus obtain a static X-ray tomogram of a knitted cotton sample at the end of wicking and in constant contact with a water solution reservoir, as seen in Fig. 2. In the 


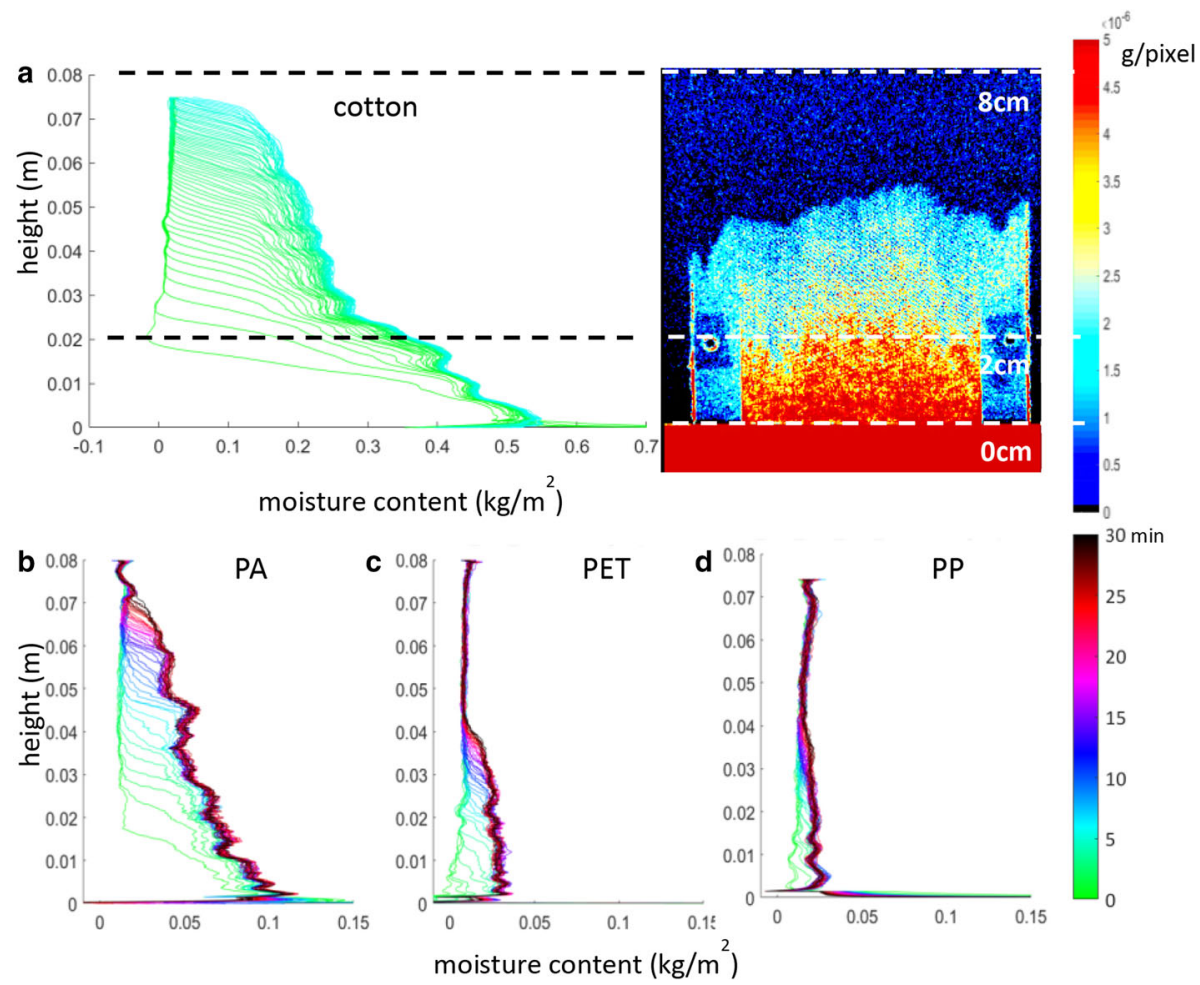

Fig. 10 a Moisture content profiles of knitted cotton sample (left) at different time steps and moisture map (right) at time $=75 \mathrm{~s}$. Bottom: Moisture content profiles for the $\mathbf{b}$ woven PA, $\mathbf{c}$ knitted PET, and $\mathbf{d}$ knitted PP samples at different time steps. Top color bar indicates moisture mass in moisture map and applies to the moisture content distribution. Lower color bar indicates time in minutes and applies to the 4 moisture content profiles versus time graphs

section view of the 3D dataset (Fig. 2c), it is clear that, at the bottom, water is present not only inside the yarns but also between them filling the inter-yarn voids. This water layer seems to stick onto the textile and appears to be thicker than the textile itself. The CT images (see Fig. 2) are an indication of this thickness. We can see in Fig. 2c that there is a meniscus at the bottom which reaches a height of $h_{m}=2.24 \mathrm{~mm}$. We can compare this height with the meniscus height on a vertical flat plate, which can be analytically calculated using the following equation (Newman and Spelt 1996):

$$
\sin \theta=1-\frac{\Delta \rho g h_{m}^{2}}{2 \gamma}
$$

where $\theta$ is the contact angle, $\Delta \rho$ the density difference between liquid and vapor phases, $g$ gravity, $h$ the meniscus height and $\gamma$ the liquid-vapor surface tension. With the measured contact angle for brown cotton with the surfactant solution of $0^{\circ}$, the theoretical meniscus height is $2.67 \mathrm{~mm}$, in good agreement with the measured height.

At the bottom of the meniscus, the width is $w_{0}=5.5 \mathrm{~mm}$. Above the meniscus, the width of the bottom water region stays relatively constant with values of $w_{25}=0.72 \mathrm{~mm}$, $w_{50}=0.64 \mathrm{~mm}, w_{75}=0.64 \mathrm{~mm}$ and $w_{100}=0.60 \mathrm{~mm}$, at heights of $25,50,75$ and $100 \%$ of maximum height $h_{\max }=19 \mathrm{~mm}$. A simplified model of water uptake for this region 

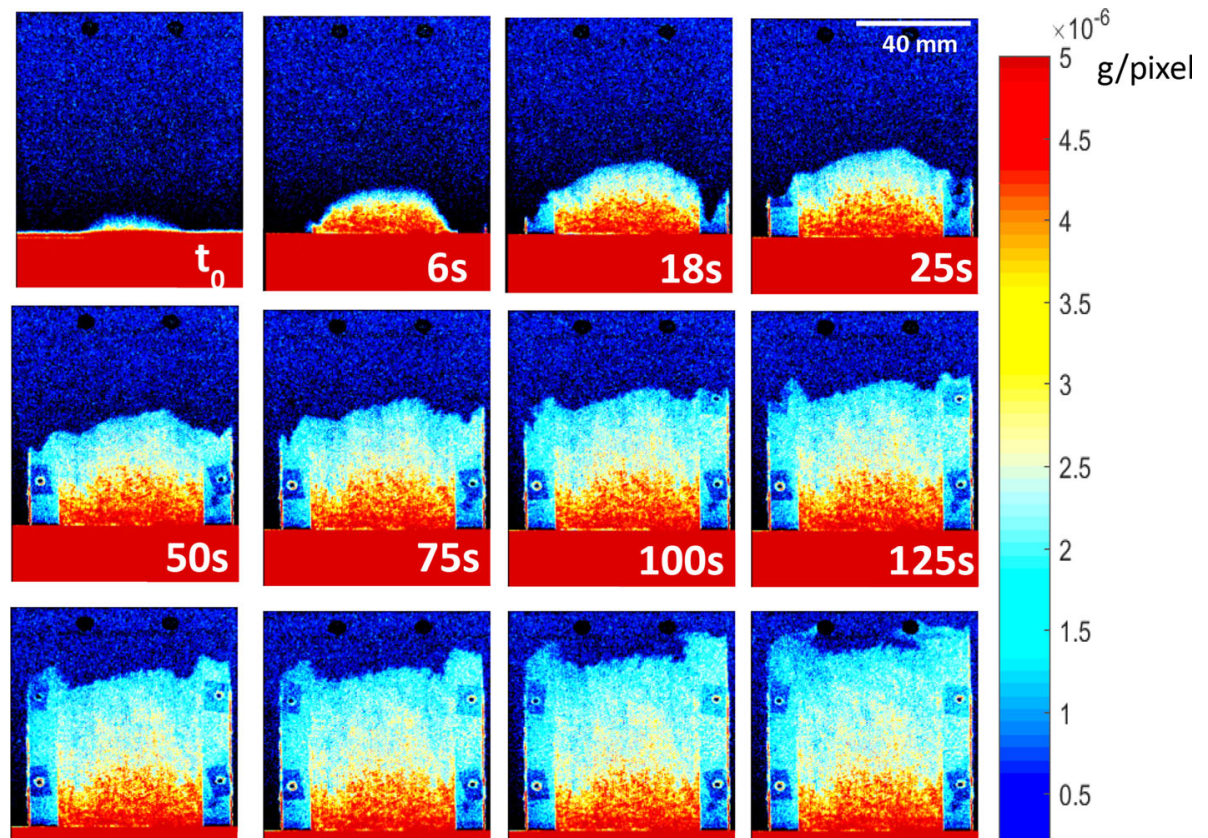

150s
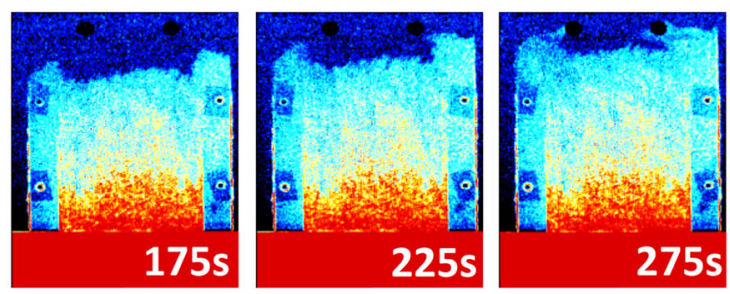

$-3$

2.5

2

1.5

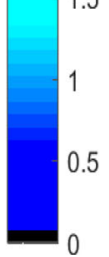

Fig. 11 Evolution of moisture content distribution in knitted cotton sample undergoing water uptake over $275 \mathrm{~s}$

considers the fabric to be a group of parallel cylinders with a water film between them. For two cylinders of the same radius, the maximum height of the liquid is given by Liu et al. (2007):

$$
h_{\max }=\frac{\gamma \cos \theta}{\Delta \rho g R}
$$

where $R$ is the circumferential radius of the curvature of the liquid-vapor interface in a horizontal cross section (see Fig. 12a).

To determine curvature $R$ on a wet sample, we do the following procedure. First, we try to find a horizontal plane in the dry sample where the yarns are mostly perpendicular to the plane. Figure $12 \mathrm{~b}$ depicts a $3 \mathrm{D}$ view of the dry sample. Making a cut view at this plane of the wet sample leads to Fig. 12c. From this view, we can estimate $R$ by measuring the curvature on the water film between two yarns, as displayed by the green arcs in Fig. 12c. The average [range] measured radius is $\bar{R}=230$ [90-546] $\mu \mathrm{m}$ resulting, according to Eq. 9, in an theoretical maximum height $h_{\max }=15.5$ [6.5-39.9] $\mathrm{mm}$. The measured value of $h_{\max }=19 \mathrm{~mm}$.

Above this lower region, no more water bridge between yarns is seen. Such microCT dataset complements the 2D neutron projections with 3D information.

We can now understand that each region has its own wetting front, where each front has its distinct wetting behavior. The lower region, where there are liquid bridges between yarns, reaches its maximum height relatively quickly due to gravity, while the top region, where there are no liquid bridges, continues to wick until the full sample is wet. The different behavior of the two fronts is studied further using moisture profiles. 

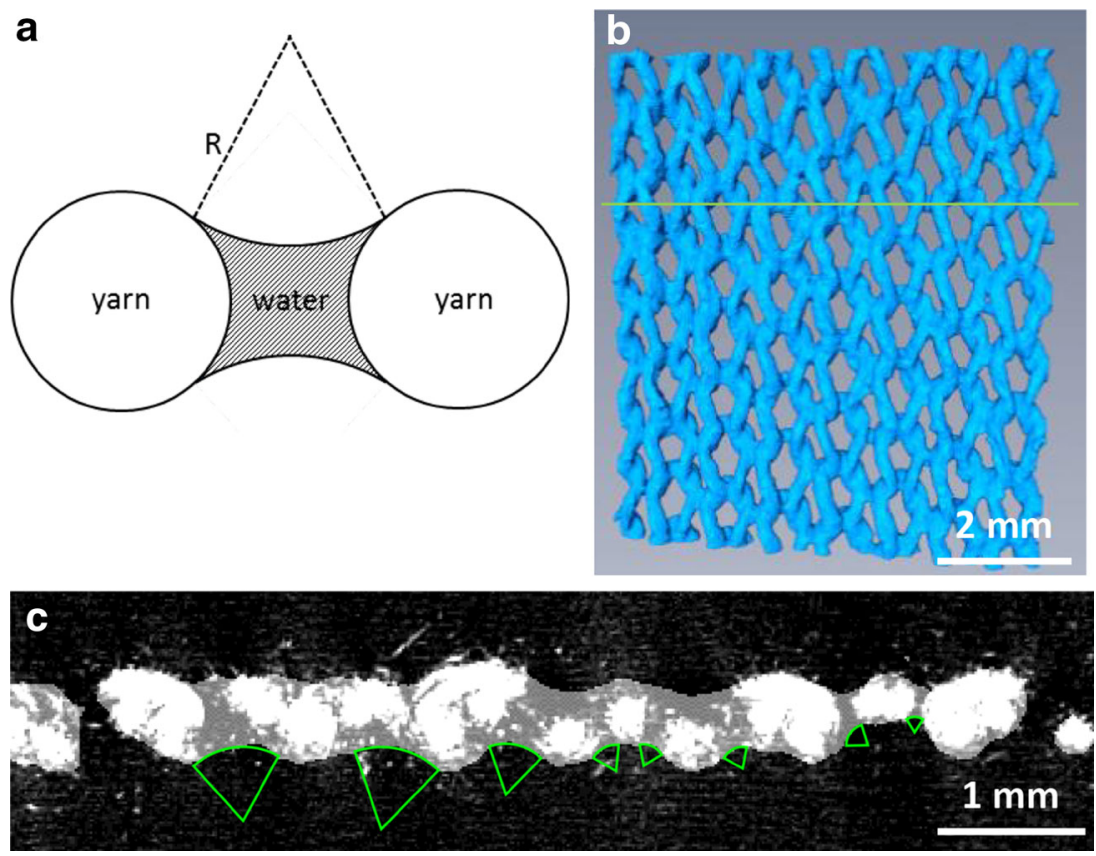

Fig. 12 a Horizontal cross section of simplified geometry considered here of liquid between two parallel cylinders. $R$ is the radius of the curvature of the liquid-vapor interface. $\mathbf{b} 3 \mathrm{D}$ view of the dry sample with a sample horizontal cross section (green). c Horizontal cross section of wet sample. Yarns are in white and water is in gray. Green arcs are measurements of $R$

\subsection{Moisture Profiles}

The difference between the behavior of the two water uptake regions is more explicitly studied by analyzing the moisture content profiles versus time, providing the time evolution of moisture content along the longitudinal middle section (Fig. 10).

Figure 10a shows the moisture content profiles along the height of a knitted cotton sample versus time. The region under around $20 \mathrm{~mm}$ has much higher moisture than the region above it, as can be seen by the yellow-to-red-colored region on the moisture map (right). Figure 10b, $\mathrm{c}$ and $\mathrm{d}$ shows the moisture content profiles at the longitudinal middle section of one sample of PA, PET and PP yarn. The height is measured from the reservoir level.

Again we see that cotton absorbs a much larger amount of moisture than the other samples and is also much faster, reaching the top in less than $10 \mathrm{~min}$. PA and PET display a behavior very different than the one of cotton. Both PA and PET appear to only have one wicking region where wicking takes much longer and the top is not reached in all samples. The PP fabric wicks very little moisture making its results poorly repeatable.

\subsection{Front Height Versus Time}

Using the image processing detailed in Sect. 4.3.1, we obtain the wicking front. A different threshold value is calculated per sample. As the analysis described below only applies to a single height, a single front has to be selected for the cotton samples. We select the highest, i.e., the intra-yarn front, for cotton. The front height is determined as the average height of 

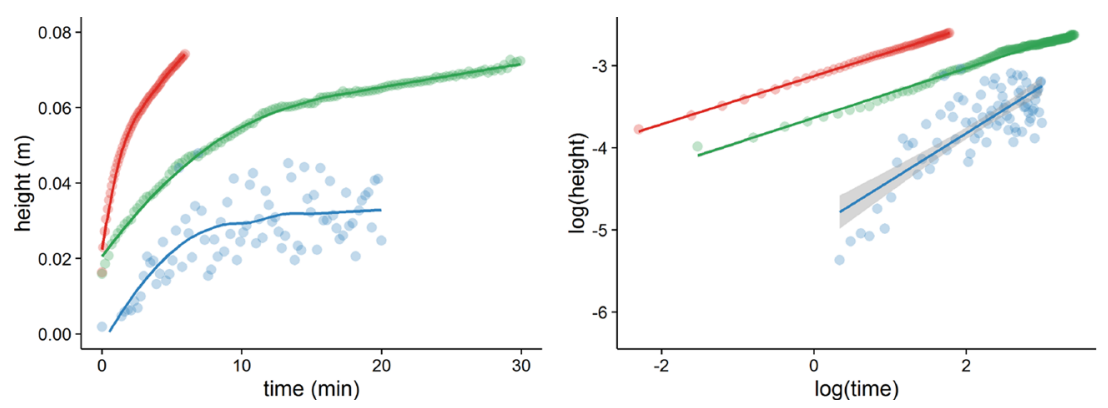

Fig. 13 Height as a function of time for some selected samples: cotton knit, PA woven, PET knit. Left: linear plot, right: logarithmic plot. Solid line represents a smoothing fit for the linear plot and a linear fit on the logarithmic plot

the front in the central part of the sample. We use 200 pixels $(21 \mathrm{~mm})$ as the averaging width. Figure 13 shows examples of curves obtained this way.

We can model the wicking behavior as a power law. Equation (10) shows the relationship between wicking height $h$ and time $t$ using the constants $A$ and $b$ of a specific fabric. The exponent $b$ is the wicking ratio and can be visualized as the slope of the function in a logarithmic plot. It would also be possible to model the behavior based on the mass (instead of height) with a similar power law; however, here we use height to be a simpler comparison to the Washburn equation (1921) and also to be able to compare with the wicking literature.

$$
h=A t^{b}
$$

For each sample, we fit a power law function to find coefficients $A$ and $b$. Table 3 contains the wicking ratio $(b)$ and wicking constant $(A)$ measured for all fabrics. There is no discernible effect of twisting level, fabric structure or wicking direction. Note that since there is almost no wicking in PP there is no useful data.

The wicking constant $A$ is a measure of the speed of wicking and is higher in cotton samples, followed by PA samples and then PET samples. This is a result of the different wettability of each material (as measured through the contact angle in Table 1). Both wicking ratio and wicking constant have large variability, specially for the synthetic materials. Furthermore, most fabrics showed a wicking ratio lower than as predicted by the Washburn equation (1921), which corresponds to a wicking ratio of 0.5. We think this can be attributed to gravity which is neglected in the Washburn equation. Last, the correlation coefficient of the fit is very good for cotton samples (higher than 0.95) and good, but not perfect, (higher than 0.70 on average) model for synthetic fibers. The main reason why the correlation coefficient is not very good for synthetic fibers is that since they wick much less water overall the relative noise in the neutron images is higher. Evaporation has been found to have negligible influence on the wicking process for the boundary conditions in the experiments.

Further observations can be made for the cotton samples based on the CT scan. There are clearly two systems of wicking. One front happens inside yarns and reaches the top of the sample. The other, which happens around the yarns, reaches its maximum height quickly around $20 \mathrm{~mm}$.

These two systems behave differently. While the wicking inside the yarns resembles a normal capillary uptake, the wicking around the yarns and on the voids resembles a film sticking around and between parallel cylinders. The geometry of the wicking volume of the lower region covers the yarns but also creates liquid bridges between yarns. The meniscus at 
Table 3 Wicking ratio for all samples across all twist levels, fabric structures and wicking directions

\begin{tabular}{|c|c|c|c|}
\hline Material & $\begin{array}{l}\text { Wicking } \\
\text { ratio (b: [1/s]) }\end{array}$ & $\begin{array}{l}\text { Wicking constant } \\
\left.\text { (A: } 10^{-2}[\mathrm{~m}]\right)\end{array}$ & Fit $\mathrm{R}^{2}$ \\
\hline Cotton (brown), 800 tpm, knit course & 0.29 & 1.33 & $1.00 *$ \\
\hline Cotton (brown), 800 tpm, knit wale & 0.43 & 0.67 & 0.99 \\
\hline Cotton (brown), 800 tpm, woven & 0.35 & 1.11 & $1.00 *$ \\
\hline Cotton (brown), 1000 tpm, knit course & 0.35 & 0.93 & $1.00 *$ \\
\hline Cotton (brown), 1000 tpm, knit wale & 0.31 & 1.19 & $1.00^{*}$ \\
\hline Cotton (brown), 1000 tpm, woven & 0.33 & 1.24 & $1.00 *$ \\
\hline Average cotton (brown) & $0.34 \pm 0.04$ & $1.08 \pm 0.22$ & \\
\hline Cotton (white), 800 tpm, knit course & 0.34 & 1.03 & $1.00 *$ \\
\hline Cotton (white), 800 tpm, knit wale & 0.41 & 0.78 & 0.98 \\
\hline Cotton (white), 800 tpm, woven & 0.33 & 1.19 & 0.99 \\
\hline Cotton (white), 1000 tpm, knit course & 0.32 & 1.11 & $1.00 *$ \\
\hline Cotton (white), 1000 tpm, knit wale & 0.34 & 1.03 & $1.00 *$ \\
\hline Cotton (white), 1000 tpm, woven & 0.31 & 1.32 & $1.00^{*}$ \\
\hline Average cotton (white) & $0.34 \pm 0.03$ & $1.08 \pm 0.16$ & \\
\hline PET, 100 tpm, knit course & 0.65 & 0.03 & 0.64 \\
\hline PET, 100 tpm, knit wale & 0.39 & 0.21 & 0.74 \\
\hline PET, 100 tpm, woven & 0.18 & 1.62 & 0.86 \\
\hline PET, 200 tpm, knit wale & 0.36 & 0.49 & 0.78 \\
\hline PET, 200 tpm, woven & 0.28 & 0.51 & 0.34 \\
\hline PET, 300 tpm, knit course & 0.25 & 0.63 & 0.85 \\
\hline PET, 300 tpm, knit wale & 0.36 & 0.39 & 0.98 \\
\hline PET, 300 tpm, woven & 0.74 & 0.03 & 0.76 \\
\hline Average PET & $0.40 \pm 0.18$ & $0.49 \pm 0.48$ & \\
\hline PA, 100 tpm, knit course & 0.27 & 0.52 & 0.63 \\
\hline PA, 100 tpm, knit wale & 0.40 & 0.25 & 0.82 \\
\hline PA, 100 tpm, woven & 0.29 & 0.82 & 0.99 \\
\hline PA, 200 tpm, knit course & 0.16 & 1.91 & 0.83 \\
\hline PA, 200 tpm, knit wale & 0.28 & 0.69 & 0.93 \\
\hline PA, 200 tpm, woven & 0.29 & 0.82 & 0.97 \\
\hline PA, 300 tpm, knit course & 0.23 & 1.05 & 0.91 \\
\hline PA, 300 tpm, knit wale & 0.52 & 0.12 & 0.90 \\
\hline PA, 300 tpm, woven & 0.35 & 0.67 & 0.93 \\
\hline Average PA & $0.31 \pm 0.10$ & $0.76 \pm 0.49$ & \\
\hline
\end{tabular}

$*>0.995$ rounded to 1.00

Averages and standard deviation for each material is also presented

the bottom is thicker similar to the meniscus on a flat vertical plate (see Newman and Spelt 1996). This film sticking to the fabric is thick, so that gravity will play an important role. The effect of gravity is larger in this lower region, but will also play a role in the top region, as evidenced by the wicking ratio below 0.5 measured above. The influence of gravity can also be seen in the logarithmic plots in Fig. 8. Without gravity effect, the logarithmic plot would be a line, but with gravity the later end of the line is curved downward (lower height 
or moisture content expected for that time). Also of note, as seen in Fig. 11, the higher region is always in front of the lower region.

\section{Conclusion}

In this paper, we show how neutron radiography can be used for the investigation of wicking in textiles. The proposed methodology allows to quantify moisture with pixel size of $100 \mu \mathrm{m}$ at temporal resolution in the order of 3-10 s. The image processing steps needed to extract the moisture content from the intensity images are presented. The removal of the scattering distortion by the reservoir is explained as well as the segmentation of the wetted areas. Finally, we show that the mass measurements obtained directly from the images match the gravimetric measurements and explore different types of analysis possible from the dataset.

We found that the full mapping of moisture content allows us to differentiate two different regions of the wicking process in the cotton samples showing that the single sharp front approximation an inadequate model for cotton. The presence of these two regions was further confirmed by the X-ray CT scan. The moisture profiles for PA and PET only show a single wicking region however. This result shows that the single sharp front approximation is adequate for low wicking fabrics.

The analysis of the wicking front behavior indicates that, at least for cotton, the power law is a good model for wicking. The wicking ratios found depend mainly on the material itself (i.e., wettability), and not on fabric parameters such as yarn twisting level, fabric structure or wicking direction. However, there is a large variability on different repeats as shown by the standard deviation. Fabrics which wick slower have a larger variability. Differences in fiber cross section could not be evaluated since the PP fabrics did not wick.

Acknowledgements This study was funded by ETH Grant \# 0-20909-13. The authors would like to thank Bäumlin \& Ernst AG, CWC Textil AG, Chemosvit Fibrochem and LEGS for providing the yarns. We thank Prof. Artan Sinoimeri, from LPMT at Université de Haute-Alsace (Moulhouse-France), for helping us with the re-twisting of cotton yarns. We also thank Stefan Hartmann from Empa X-ray analytics Center for the help with the X-ray CT scan, Stefan Carl, from Empa, for the support in developing the different experimental devices and Jan Hovind, from PSI, for support during neutron radiography experiments. We thank Lukasz Pawlicki for the production of Fig. 1c.

\section{References}

Birrfelder, P., et al.: Effect of fiber count and knit structure on intra- and inter-yarn transport of liquid water. Text. Res. J. 83(14), 1477-1488 (2013)

Campbell, C., Parish, L.C.: The decubitus ulcer: facts and controversies. Clin. Dermatol. 28(5), 527-532 (2010)

Derler, S., et al.: Medical textiles with low friction for decubitus prevention. Tribol. Int. 46(1), 208-214 (2012)

Derluyn, H., et al.: Characterizing saline uptake and salt distributions in porous limestone with neutron radiography and X-ray micro-tomography. J. Build. Phys. 36(4), 353-374 (2013)

Eller, J., et al.: Progress in In Situ X-Ray Tomographic Microscopy of Liquid Water in Gas Diffusion Layers of PEFC. J. Electrochem. Soc. 158(8), B963 (2011)

Hassanein, R.: Correction methods for the quantitative evaluation of thermal neutron tomography. Doctoral dissertation, ETH Zurich (2006)

$\mathrm{Hu}$, J., et al.: Moisture management tester: a method to characterize fabric liquid moisture management properties. Text. Res. J. 75(1), 57-62 (2005)

Keiser, C., Wyss, P., Rossi, R.M.: Analysis of steam formation and migration in firefighters' protective clothing using X-ray radiography. Int. J. Occup. Saf. Ergon.: JOSE 16(2), 217-229 (2010) 
Lee, J.H., et al.: Determining the absorption properties of split-type microfiber fabrics by measuring the change in color depth. Text. Res. J. 74(3), 271-278 (2004)

Lehmann, E.H.: Recent improvements in the methodology. PRAMANA - J. Phys. 71(4), 653-661 (2008)

Lehmann, E.H., Vontobel, P., Kardjilov, N.: Hydrogen distribution measurements by neutrons. Appl. Radiat. Isot. 61(4), 503-509 (2004)

Lehmann, E.H., Vontobel, P., Wiezel, L.: Properties of the radiography facility neutra at Sin $q$ and its potential for use as European Reference Facility. Nondestruct. Test. Eval. 16(2-6), 191-202 (2001)

Liu, T., Choi, K.F., Li, Y.: Capillary rise between cylinders. J. Phys. D Appl. Phys. 40, 5006-5012 (2007)

Masoodi, R., Pillai, K. (eds.): Wicking in porous materials: traditional and modern modeling approaches. CRC Press, Boca Raton (2012)

Mittal, K.L.: Determination of CMC of polysorbate 20 in aqueous solution by surface tension method. J. Pharm. Sci. 61(8), 1334-1335 (1972)

Newman, A.W., Spelt, J.K. (eds.): Applied surface thermodynamics. CRC Press, Boca Raton (1996)

Niedermann, R., Rossi, R.M.: Objective and subjective evaluation of the human thermal sensation of wet fabrics. Text. Res. J. 82(4), 374-384 (2012)

Niño, M.R.R., Patino, J.M.R.: Surface tension of bovine serum albumin and tween 20 at the air-aqueous interface. J. Am. Oil. Chem. Soc. 75(10), 1241-1248 (1998)

Parada, M., et al.: A review on advanced imaging technologies for the quantification of wicking in textiles. Text. Res. J. 87(1), 110-132 (2017)

Pleinert, H., Lehmann, E.: Determination of hydrogenous distributions by neutron transmission analysis. Physica B 234-236, 1030-1032 (1997)

Raja, D., et al.: Comparison of different methods to measure the transverse wicking behaviour of fabrics. J. Ind. Text. 43(3), 366-382 (2014)

Reifler, F.a: The method of neutron imaging as a tool for the study of the dynamics of water movement in wet aramid-based ballistic body armour panels. Meas. Sci. Technol. 17(7), 1925-1934 (2006)

Rotaru, G.M., et al.: Friction between human skin and medical textiles for decubitus prevention. Tribol. Int. 65, 91-96 (2013)

Savitzky, A., Golay, M.J.E.: Smoothing and differentiation of data. Anal. Chem. 36(8), 1627-1639 (1964)

Schafer, R.W.: What is a Savitzky-Golay filter? IEEE Signal Process. Mag. 28(4), 111-117 (2011)

Schindelin, J., et al.: Fiji: an open-source platform for biological-image analysis. Nat. Methods 9(7), 676-682 (2012)

Sedighi-Gilani, M., Vontobel, P., Lehmann, E., et al.: Liquid uptake in Scots pine sapwood and hardwood visualized and quantified by neutron radiography. Mater. Struct. 47(6), 1083-1096 (2014)

Sedighi-Gilani, M., et al.: Visualization and quantification of liquid water transport in softwood by means of neutron radiography. Int. J. Heat Mass Transf. 55(21-22), 6211-6221 (2012)

Taheri, M., Vadood, M., Johari, M.S.: Investigating the effect of yarn count and twist factor on the packing density and wicking height of lyocell ring-spun yarns. Fibers Polym. 14(9), 1548-1555 (2013)

Vatansever, F., et al.: Toward fabric-based flexible microfluidic devices: pointed surface modification for $\mathrm{pH}$ sensitive liquid transport. ACS Appl. Mater. Interfaces 4(9), 4541-4548 (2012)

Wardiningsih, W., Troynikov, O.: Influence of cover factor on liquid moisture transport performance of bamboo knitted fabrics. J. Text. Inst. 103(1), 89-98 (2012)

Washburn, E.W.: The dynamics of capillary flow. Phys. Rev. 17, 273-283 (1921)

Weder, M., Brühwiler, P.a, Laib, A.: X-ray tomography measurements of the moisture distribution in multilayered clothing systems. Text. Res. J. 76(1), 18-26 (2006)

Yao, B., et al.: An improved test method for characterizing the dynamic liquid moisture transfer in porous polymeric materials. Polym. Test. 25(5), 677-689 (2006)

Zhu, C., Takatera, M.: A new thermocouple technique for the precise measurement of in-plane capillary water flow within fabrics. Text. Res. J. 84(5), 513-526 (2014)

Zhu, C., Takatera, M.: Effects of hydrophobic yarns on liquid migration in woven fabrics. Text. Res. J. 85(5), 479-486 (2015) 